\title{
The efficacy and safety of CapeOX plus bevacizumab therapy followed by capecitabine plus bevacizumab maintenance therapy in patients with metastatic colorectal cancer: a multi-center, single-arm, phase II study (CCOG-0902)
}

Goro Nakayama ${ }^{1 *}$, Kiyoshi Ishigure ${ }^{2}$, Hiroyuki Yokoyama ${ }^{3}$, Keisuke Uehara ${ }^{4}$, Hiroshi Kojima ${ }^{5}$, Akiharu Ishiyama ${ }^{6}$, Naomi Hayashi', Nao Takano ${ }^{1}$, Norifumi Hattori ${ }^{1}$, Daisuke Kobayashi ${ }^{1}$, Chie Tanaka ${ }^{1}$, Masamichi Hayashi ${ }^{1}$, Mitsuro Kanda ${ }^{1}$, Suguru Yamada ${ }^{1}$, Hiroyuki Sugimoto ${ }^{1}$, Masahiko Koike ${ }^{1}$, Michitaka Fujiwara ${ }^{1}$, Tsutomu Fujii' Kenta Murotani ${ }^{8}$, Yuichi Ando ${ }^{9}$ and Yasuhiro Kodera ${ }^{1}$

\begin{abstract}
Background: The aim of this study was to evaluate the efficacy and safety of CapeOX plus bevacizumab with a planned oxaliplatin stop-and-go strategy in Japanese patients with metastatic colorectal cancer (mCRC).

Methods: Patients with untreated mCRC were treated with 4 cycles of CapeOX plus bevacizumab therapy, followed by capecitabine plus bevacizumab maintenance therapy. Reintroduction of oxaliplatin was scheduled after 8 cycles of maintenance therapy or upon tumor progression. The primary endpoint was progression-free survival (PFS), and secondary end points included overall survival (OS), objective response rate to each treatment, reintroduction rate of oxaliplatin, frequency of peripheral sensory neuropathy (PSN), and safety.

Results: The 52 patients who received the protocol treatment were included in the evaluation of efficacy and safety. Median PFS and OS were 12.4 months (95\% confidence interval [Cl], 10.0-14.8) and 30.6 months (95\% Cl, 27. 6-33.5), respectively. The objective response rates were 55.8\% for the initial CapeOX plus bevacizumab therapy, 17. $8 \%$ for capecitabine plus bevacizumab maintenance therapy, and 31.0\% for reintroduced CapeOX plus bevacizumab therapy. The frequency of PSN was $63.5 \%$, including $3.8 \%$ of patients with grade 3 PSN. No patients required treatment discontinuation because of PSN during the induction or maintenance therapy.
\end{abstract}

Conclusions: CapeOX plus bevacizumab therapy with a planned oxaliplatin stop-and-go strategy is a feasible first-line treatment for Japanese patients with mCRC.

Trial registration: This trial is registered with the University Hospital Medical Information Network in 15 March 2010 (UMIN000006478).

Keywords: Metastatic colorectal cancer, Reintroduction of oxaliplatin, Maintenance therapy, Capecitabin, Bevacizumab, Peripheral sensory neuropathy

\footnotetext{
* Correspondence: goro@med.nagoya-u.ac.jp

${ }^{1}$ Department of Gastroenterological Surgery, Nagoya University Graduate

School of Medicine, 65 Tsurumai-cho, Showa-ku, Nagoya 466-8550, Japan

Full list of author information is available at the end of the article
} 


\section{Background}

The first-line treatments for patients with metastatic colorectal cancer (mCRC) usually involves combination chemotherapies that include infusional 5-fluorouracil and leucovorin plus either irinotecan or oxaliplatin $[1,2]$. Capecitabine, an oral fluoropyrimidine anticancer agent, in combination with oxaliplatin (CapeOX), had similar efficacy to regimens based on infusional 5-furuorouracil in combination with oxaliplatin used in previous studies [3-5]. The addition of bevacizumab, a humanized monoclonal antibody that inhibits vascular endothelial growth factor, to chemotherapy regimens that include CapeOX improves overall survival (OS) or progressionfree survival (PFS) [5-7].

Peripheral sensory neuropathy (PSN), a cumulative dose-limiting toxicity of oxaliplatin, often requires the discontinuation of oxaliplatin before disease progression, and decreases patients' quality of life. The results of several prospective studies have suggested the intermittent use of oxaliplatin to avoid its cumulative toxicity and prolong the time to treatment failure [8-11]. However, reports that describe details of the planned oxaliplatin stop-and-go strategy in bevacizumab containing regimens are limited [12-14].

The objectives of this study were (1) to evaluate the efficacy of CapeOX plus bevacizumab therapy with planned short-term initial CapeOX plus bevacizumab therapy, followed by 8 cycles of fixed-term maintenance therapy, and then the reintroduction of oxaliplatin for patients with mCRC, and (2) to assess the safety, including the incidence of PSN, of this strategy.

\section{Methods}

\section{Study design and patients eligibility}

This multicenter, single-arm, phase 2 trial was conducted by the Chubu Clinical Oncology Group (CCOG) in 18 hospitals of Japan. The criteria for inclusion in this study were age at least 20 years; histologically proven adenocarcinoma of the colon or rectum; unresectable metastasis; no previous chemotherapy for metastatic disease; at least one measurable lesion according to the Response Evaluation Criteria in Solid Tumors (RECIST), version 1.1; an Eastern Cooperative Oncology Group (ECOG) Performance Status of 0 or 1 ; and adequate bone marrow, liver, and renal functions. Patients may register if they have received adjuvant chemotherapy with fluoropyrimidine and cancer recurrence has occurred 6 months or more after the last dose. Patients who have received oxaliplatin-based adjuvant chemotherapy may not register. Patients with brain metastasis, clinically significant cardiovascular disease, second malignancies, bowel obstruction, PNS more than grade 1 , uncontrolled diabetes mellitus or hypertension were excluded. Patients completed written informed consent before participating, and the ethics committees of Nagoya University Hospital and each participating facility approved the study. This trial was registered with the University Hospital Medical Information Network in 15 March 2010 (UMIN000006478).

\section{Treatment plan}

Induction therapy: Patients received CapeOX plus bevacizumab therapy for 4 cycles, which consisted of intravenous oxaliplatin $\left(130 \mathrm{mg} / \mathrm{m}^{2}\right)$ and bevacizumab $(7.5 \mathrm{mg} / \mathrm{kg})$ on day 1 in combination with oral capecitabine $\left(1000 \mathrm{mg} / \mathrm{m}^{2}\right.$ twice daily) given as intermittent treatment for 14 days followed by 7 days treatment-free interval, every 3 weeks. Maintenance therapy: Capecitabine plus bevacizumab therapy which consisted of intravenous administration of bevacizumab $(7.5 \mathrm{mg} / \mathrm{kg})$ on day 1 and capecitabine $\left(1000 \mathrm{mg} / \mathrm{m}^{2}\right.$ twice daily), was initiated for patients with stable disease or a superior response after 4 cycles of the induction therapy. Reintroduction therapy: Reintroduction of oxaliplatin was scheduled after 8 cycles of the maintenance therapy. Oxaliplatin was also reintroduced in the event of tumor progression before 8 cycles of the maintenance therapy (in a case where tumor progression was observed before 8 cycles of the maintenance therapy). The reintroduction therapy continued until disease progression, unacceptable toxicity, or the patient refusal.

\section{Measurements}

The primary objective of this study was PFS, defined as the time from the date therapy was initiated until the date of disease progression or death from any cause. The secondary objectives were the following: OS, defined as the time from the date that therapy was initiated until the date of death from to any cause; duration of disease control (DDC), defined as PFS in the patients without disease progression before oxaliplatin reintroduction or the patients with progression at the first evaluation after oxaliplatin reintroduction. In the case achieved disease progression before oxaliplatin reintroduction and tumor response or stabilization at the first evaluation after oxaliplatin reintroduction, DDC was defined as the sum of the initial PFS and the PFS of the reintroduction [8]; overall response rate (ORR), defined as the proportion of patients whose best response was complete response (CR) or partial response (PR); disease control rate (DCR), defined as the proportion of patients whose best response was CR, PR or stable disease (SD); reintroduction rate of oxaliplatin, defined as the proportion of patients who received the reintroduced CapeOX plus bevacizumab therapy; and the incidence of adverse events, including the frequency and severity of PSN. Tumor size and response according to 
RECIST, version 1.1, based on chest-to-pelvic region computed tomography (CT) once every 8 weeks were evaluated by the local review in each participating facility. Adverse events were assessed using National Cancer Institute Common Toxicity Criteria (NCICTC), version 3.0.

\section{Statistical analysis}

A power analysis was conducted before the study. Assuming that the threshold for PFS was 7.2 months and the expected PFS was 10.4 months, referring to data from the previous clinical trials $[4,7]$, with the enrollment period of 2 years and the follow-up period of 3 years, 47 patients were required to ensure an alpha level of 0.05 (one-sided) and a detection power (1- $\beta$ ) of $80 \%$. The sample size for this study was 50 to account for possible loss to follow-up.

The PFS, the primary objective of this study, was estimated using the Kaplan-Meier method, and the median PFS and its 95\% confidence interval were estimated. Other time-to-endpoint variables, DDC and OS, were estimated using the same method. The ORR, DCR and incidence the toxicities were calculated as proportions with exact confidence intervals. Statistical analyses were performed using SPSS, version 23 (SPSS Inc., Chicago, IL, USA).

\section{Results}

\section{Patient characteristics}

Fifty-four patients from 18 institutions were enrolled in this study between April 2010 and October 2011. Two patients were excluded after enrollment because of ineligibility. The remaining 52 patients who received the protocol treatment were included in the evaluation of efficacy and safety. Baseline characteristics of the 52 patients are presented in Table 1 .

\section{Treatment status}

The initial CapeOX plus bevacizumab therapy was administered to 52 patients, including 50 patients who accomplish 4 cycles of the induction therapy. The maintenance therapy, capecitabin and bevacizumab, was administered to 45 patients (86.5\%). Twenty-two of those patients could accomplish 8 cycles of maintenance therapy. Oxaliplatine was reintroduced in 29 patients (55.8\%), including 20 patients accomplished 8 cycles of the maintenance therapy and 9 patients with disease progression during the maintenance therapy. A consort chart of patients is presented in Fig. 1. The median number of treatment cycles was 4 (1-4 cycles) for the initial CapeOX plus bevacizumab therapy, 7 (1-8 cycles) for the capecitabin plus bevacizumab maintenance therapy, and 5 (1-22 cycles) for the reintroduced CapeOX plus bevacizumab therapy. The median time-to-
Table 1 Characteristics of patients

\begin{tabular}{|c|c|c|}
\hline \multirow[t]{2}{*}{ Variable } & \multicolumn{2}{|l|}{$N=52$} \\
\hline & $\mathrm{n}$ & $\%$ \\
\hline \multicolumn{3}{|l|}{ Sex } \\
\hline Male & 31 & 59.6 \\
\hline Female & 21 & 40.4 \\
\hline \multicolumn{3}{|l|}{ Age, years } \\
\hline Median (range) & $66(40-80)$ & \\
\hline \multicolumn{3}{|l|}{ Performance status WHO } \\
\hline 0 & 38 & 73.1 \\
\hline 1 & 14 & 26.9 \\
\hline \multicolumn{3}{|l|}{ Primary site } \\
\hline Colon & 28 & 53.8 \\
\hline Rectum & 24 & 46.2 \\
\hline \multicolumn{3}{|l|}{ Metastases } \\
\hline Synchronous & 18 & 34.6 \\
\hline Metachronous & 34 & 65.4 \\
\hline \multicolumn{3}{|l|}{ Nunmer of metastatic site } \\
\hline 1 & 37 & 71.2 \\
\hline$>1$ & 15 & 28.8 \\
\hline \multicolumn{3}{|l|}{ Metastaic sites } \\
\hline Liver & 30 & 57.7 \\
\hline Liver only & 16 & 30.8 \\
\hline Lung & 20 & 38.5 \\
\hline Peritoneum & 9 & 9.6 \\
\hline Lymph nodes & 5 & 17.3 \\
\hline \multicolumn{3}{|l|}{ Prior treatment } \\
\hline Adjuvant chemotherapy ${ }^{a}$ & 20 & 38.5 \\
\hline Surgery ${ }^{b}$ & 35 & 67.3 \\
\hline Radiotheray & 0 & 0 \\
\hline \multicolumn{3}{|l|}{ KRAS status } \\
\hline Wild type & $24 / 43$ & 55.8 \\
\hline Mutant type & $19 / 43$ & 44.2 \\
\hline
\end{tabular}

$N$ total number of patients, $n$ number of patients, $W H O$ World Health Organization

${ }^{a}$ Chemotherapy with fluoropyrimidine. No patients received oxaliplatin-based adjuvant chemotherapy

${ }^{\mathrm{b}}$ Resection of primary site

treatment failure of the protocol treatment was 9.9 months $(95 \%$ confidence interval $[\mathrm{CI}]$ : $5.9-$ 13.8 months).

The relative dose intensity of oxaliplatin in the initial and reintroduced CapeOX plus bevacizumab therapy were 92.3 and $78.5 \%$, respectively, and the cumulative dose of oxaliplatin during the overall treatment period was $1052 \mathrm{mg}(470-4346 \mathrm{mg})$. The protocol treatment was discontinued because of the following circumstances: disease progression in 28 patients (53.8\%), 


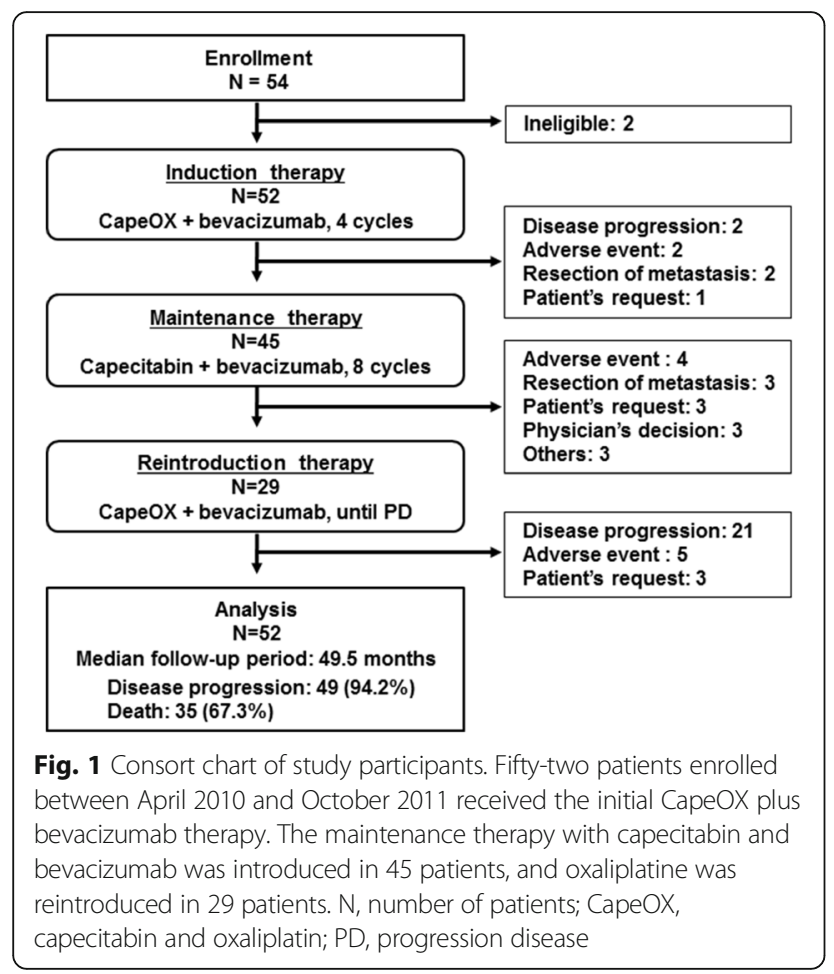

adverse events in 11 (21.2\%), and resection of metastasis in $5(9.6 \%)$.

Treatment status is summarized in Table 2.

\section{Treatment outcomes}

After a median follow-up period of 49.5 months (range: 6.3-63.9 months), the disease progressed in 49 patients
(94.2\%) and 35 deaths (67.3\%) occurred in the 52 patients enrolled. Median PFS, the primary endpoint, and DDC were 12.4 months (95\% CI, 10.0-14.8 months) and 13.4 months (95\% CI, 12.2-14.6 months), respectively (Fig. 2a). There was no significant difference between these two survival outcomes (HR 0.932, 95\% CI 0.627-1.385, $p=0.727)$. The median OS was 30.6 months (95\% CI, 27.6-33.5 months) (Fig. 2b). The ORR for the initial CapeOX plus bevacizumab therapy, maintenance capecitabin plus bevacizumab therapy, and reintroduced CapeOX plus bevacizumab therapy were $55.8,17.8$, and $31.0 \%$, respectively. The DCR for those therapies were 96.2, 80.0, and $89.7 \%$, respectively. The tumor response to each phase of treatment is summarized in Table 3.

\section{Adverse events}

The incidence of treatment-related adverse events is presented in Table 4. The frequencies of hematological and non-hematological adverse events above grade three were 13.5 and $38.5 \%$, respectively, for the overall treatment period. PSN occurred in 33 patients (63.5\%), including two patients (3.8\%) with PSN grade 3. Grade-3 PSN occurred in one patient at the end of the induction therapy and one patient after fourth cycle of the reintroduction therapy. The frequencies of PNS after 4 cycles of the initial CapeOX plus bevacizumab therapy and after 8 cycles of the maintenance therapy were 42 and $9 \%$, respectively (Fig. 3). During the induction and maintenance therapy, no patients required treatment discontinuation due to PSN. The incidence of hand-foot

Table 2 Treatment status of patients

\begin{tabular}{|c|c|c|c|}
\hline & $\begin{array}{l}\text { Induction therapy } \\
\text { CapeOX }+ \text { BEV } \\
(N=52)\end{array}$ & $\begin{array}{l}\text { Maintenance therapy } \\
\text { Capecitabin + BEV } \\
(N=45)\end{array}$ & $\begin{array}{l}\text { Reintoroduction therapy } \\
\text { CapeOX }+ \text { BEV } \\
(N=29)\end{array}$ \\
\hline \multicolumn{4}{|l|}{ Treatment cycle, times } \\
\hline Median (range) & $4(2-4)$ & $7(1-8)$ & $5(1-21)$ \\
\hline \multicolumn{4}{|c|}{ Median relative dose-intensity, \% } \\
\hline Oxaliplatin & 92.3 & - & 78.5 \\
\hline Capecitabin & 92.0 & 82.4 & 71.8 \\
\hline Bevacizumab & 96.6 & 92.0 & 91.0 \\
\hline \multicolumn{4}{|l|}{ Total dose of oxaliplatin, mg } \\
\hline Median (range) & $1052(470-4346)$ & & \\
\hline \multicolumn{4}{|c|}{ Time-to-treatment failuer, months } \\
\hline Median (95\% Cl) & $9.9(5.9-13.8)$ & & \\
\hline \multicolumn{4}{|c|}{ Post progression treatment, n (\%) } \\
\hline Second-line therapy & 46 & $(88.5)$ & \\
\hline Anti-EGFR agents & 17 & $(32.7)$ & \\
\hline Bevacizumab (BBP) & 35 & $(67.3)$ & \\
\hline Resection of metastasis & 8 & $(15.4)$ & \\
\hline
\end{tabular}




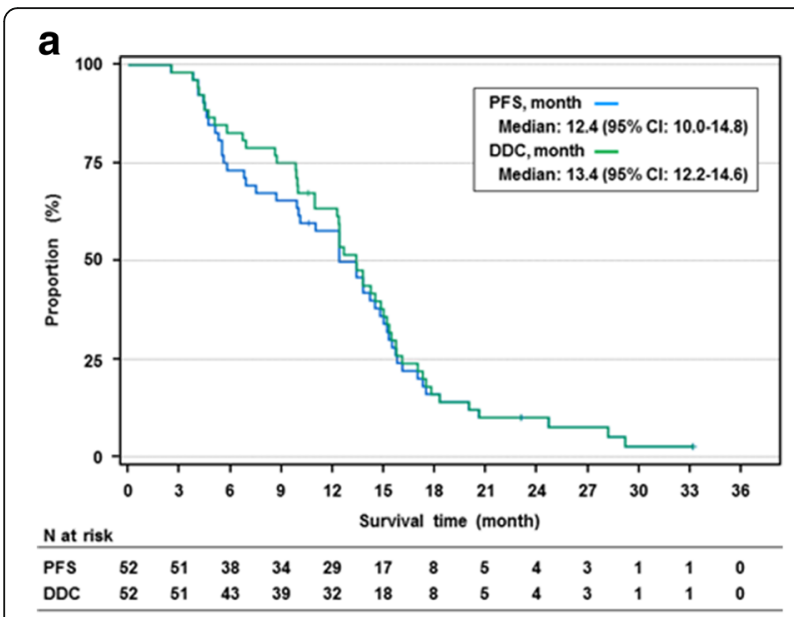

b

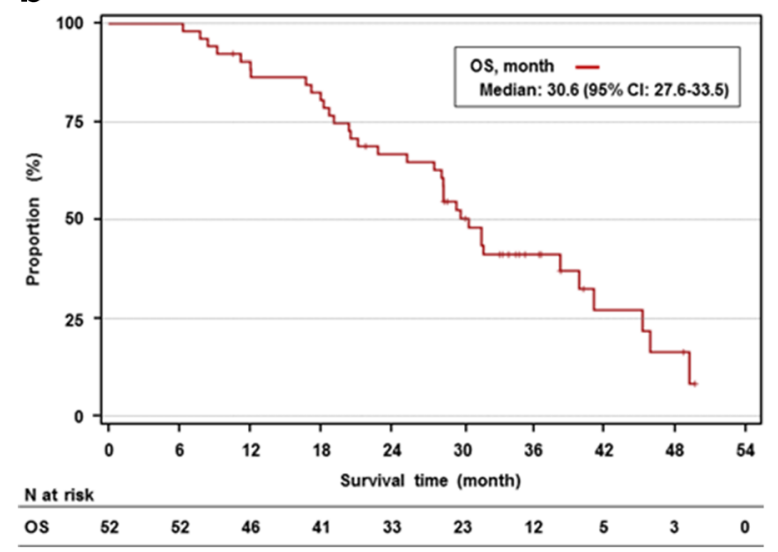

Fig. 2 a. Kaplan-Meier analysis of progression-free survival and duration of disease control. Median progression-free survival and duration of disease control were 12.4 months (95\% Cl: 10.0-14.8 months) and 13.4 months (95\% Cl: 12.2-14.6 months), respectively. PFS, progression-free survival; $\mathrm{DDC}$, duration of disease control; $\mathrm{Cl}$, confidence interval; $\mathrm{N}$, number of patients. b. Kaplan-Meier analysis of overall sunvival. Median overall survival was 30.6 months (95\% Cl: 27.6-33.5 months). OS, overall sunvival, OS; Cl, confidence interval; $\mathrm{N}$, number of patients syndrome and bevacizumab-related toxicities is presented in Fig. 3a and b, respectively. These toxicities occurred in relatively constant rate of patients throughout all treatment period, however, treatment was discontinued in 3 patients $(5.8 \%)$ due to HFS and 4 patients $(7.7 \%)$ due to bevacizumab-related toxicities.

\section{Discussion}

The present phase II study was conducted to evaluate the efficacy and safety of CapeOX plus bevacizumab with a planned stop-and-go strategy as the first-line setting in Japanese patients with mCRC. The median PFS, the primary endpoint, was 12.4 months, which was considered acceptable according to the formulation of our hypothesis that assumes the threshold and expected survival time of 7.2 months and 10.4 months, respectively. Furthermore, the other survival outcomes including OS of 30.6 months and DCC of 13.4 months were comparable to those reported in several studies evaluating oxaliplatin-based chemotherapies plus bevacizumab with intermittent use of oxaliplatin [12-14].

Fifty percent of our patients experienced PSN during initial CapeOX plus bevacizumab therapy, with a median cumulative oxaliplatin dose of $782 \mathrm{mg}$. However, after 8 cycles of maintenance therapy, the frequency of PSN decreased to $9.1 \%$. No patients required treatment discontinuation due to PSN during the initial CapeOX plus bevacizumab therapy or the maintenance therapy. In addition, severe PSN of grade 3 or higher was observed in only $3.8 \%$ of patients throughout the treatment period. These results were comparable to those from previous studies $[8,12-14]$, and might have contributed to the longer time-to-treatment failure of 9.9 months in our current study.

The design of our treatment protocol included a brief induction therapy of 4 cycles with CapeOX plus bevacizumab followed by fixed-term maintenance therapy of $8 \mathrm{cy}$ cles with capecitabin plus bevacizumab. This differed from those in previously reported studies, which mostly included 6 cycles of induction therapy followed by

Table 3 Objective tumor response

\begin{tabular}{|c|c|c|c|c|c|c|}
\hline & \multicolumn{2}{|c|}{$\begin{array}{l}\text { Induction therapy } \\
\text { CapeOX + BEV } \\
(N=52)\end{array}$} & \multicolumn{2}{|c|}{$\begin{array}{l}\text { Maintenance therapy } \\
\text { Capecitabin + BEV } \\
(N=45) \\
\end{array}$} & \multicolumn{2}{|c|}{$\begin{array}{l}\text { Reintoroduction therapy } \\
\text { CapeOX + BEV } \\
(N=29) \\
\end{array}$} \\
\hline & $n$ & $\%$ & $n$ & $\%$ & $n$ & $\%$ \\
\hline$C R$ & 0 & 0 & 0 & 0 & 2 & 6.9 \\
\hline PR & 29 & 55.8 & 8 & 17.8 & 7 & 24.1 \\
\hline SD & 21 & 40.4 & 28 & 62.2 & 17 & 58.6 \\
\hline PD & 2 & 3.8 & 9 & 20.0 & 3 & 10.3 \\
\hline ORR, \% & 55.8 & & 17.8 & & 31.0 & \\
\hline DCR, \% & 96.2 & & 80.0 & & 89.7 & \\
\hline
\end{tabular}

$B E V$ bevacizumab, $N$ total number of patients, $n$ number of patients, $C R$ complete response, $P R$ partial response, $S D$ stable disease, $P D$ progressive disease, $O R R$ objective response rate $([C R+P R] / N \times 100), D C R$ disease control rate $([C R+P R+S D] / N \times 100)$ 
Table 4 Occurrence of common toxicities

\begin{tabular}{|c|c|c|c|c|c|c|c|c|c|c|c|c|}
\hline \multirow[t]{3}{*}{ Toxicity } & \multicolumn{4}{|c|}{$\begin{array}{l}\text { Induction therapy } \\
\text { CapeOX + BEV } \\
(N=52)\end{array}$} & \multicolumn{4}{|c|}{$\begin{array}{l}\text { Maintenance therapy } \\
\text { Capecitabin + BEV } \\
(N=45)\end{array}$} & \multicolumn{4}{|c|}{$\begin{array}{l}\text { Reintoroduction therapy } \\
\text { CapeOX + BEV } \\
(N=29)\end{array}$} \\
\hline & \multicolumn{2}{|c|}{ All grade } & \multicolumn{2}{|c|}{$\geq$ Grade 3} & \multicolumn{2}{|c|}{ All grade } & \multicolumn{2}{|c|}{$\geq$ Grade 3} & \multicolumn{2}{|c|}{ All grade } & \multicolumn{2}{|c|}{$\geq$ Grade 3} \\
\hline & $n$ & $\%$ & $n$ & $\%$ & $n$ & $\%$ & $n$ & $\%$ & $n$ & $\%$ & $n$ & $\%$ \\
\hline Hematologic toxiciy & 20 & 38.5 & 5 & 9.6 & 12 & 26.7 & 3 & 6.7 & 11 & 37.9 & 2 & 6.9 \\
\hline Neutropenia & 10 & 19.2 & 4 & 7.7 & 4 & 8.9 & 2 & 4.4 & 4 & 13.8 & 2 & 6.9 \\
\hline Thrombocytopenia & 9 & 17.3 & 0 & 0 & 8 & 17.8 & 1 & 2.2 & 8 & 27.6 & 0 & 0 \\
\hline Anemia & 9 & 17.3 & 0 & 0 & 4 & 8.9 & 0 & 0 & 3 & 7.1 & 0 & 0 \\
\hline Febrile neutropenia & 1 & 1.9 & 1 & 1.9 & 0 & 0 & 0 & 0 & 0 & 0 & 0 & 0 \\
\hline Non-hematologic toxicity & 44 & 84.6 & 8 & 15.4 & 36 & 80.0 & 6 & 13.3 & 22 & 75.9 & 6 & 20.7 \\
\hline Diarrhea & 7 & 13.5 & 1 & 1.9 & 2 & 4.4 & 0 & 0 & 2 & 6.9 & 0 & 0 \\
\hline Nausea/vomiting & 8 & 15.4 & 0 & 0 & 2 & 4.4 & 0 & 0 & 3 & 10.3 & 0 & 0 \\
\hline Mucositis & 8 & 15.4 & 0 & 0 & 2 & 4.4 & 0 & 0 & 2 & 6.9 & 0 & 0 \\
\hline Hand-foot syndrome & 33 & 63.5 & 1 & 1.9 & 22 & 48.9 & 4 & 8.9 & 16 & 55.2 & 3 & 10.3 \\
\hline Fatigue & 0 & 0 & 0 & 0 & 1 & 2.2 & 0 & 0 & 1 & 3.4 & 0 & 0 \\
\hline Peripheral neuropathy & 26 & 50.0 & 1 & 1.9 & 17 & 37.8 & 0 & 0 & 14 & 48.3 & 2 & 6.9 \\
\hline Allergy & 2 & 3.8 & 1 & 1.9 & 0 & 0 & 0 & 0 & 3 & 10.3 & 2 & 6.9 \\
\hline BEV-related toxicities & 14 & 26.9 & 1 & 1.9 & 17 & 37.8 & 1 & 2.2 & 11 & 37.9 & 1 & 3.4 \\
\hline Hypertension & 9 & 17.3 & 0 & 0 & 11 & 24.4 & 0 & 0 & 8 & 27.6 & 0 & 0 \\
\hline Proteinuria & 6 & 11.5 & 0 & 0 & 5 & 11.1 & 0 & 0 & 3 & 10.3 & 1 & 3.4 \\
\hline Bleeding & 0 & 0 & 0 & 0 & 0 & 0 & 0 & 0 & 1 & 3.4 & 0 & 0 \\
\hline Infection & 1 & 1.9 & 0 & 0 & 2 & 4.4 & 1 & 2.2 & 1 & 3.4 & 0 & 0 \\
\hline Thrombosis & 1 & 1.9 & 1 & 1.9 & 0 & 0 & 0 & 0 & 0 & 0 & 0 & 0 \\
\hline
\end{tabular}

$B E V$ bevacizumab, $N$ total number of patients, $n$ number of patients

maintenance therapy until disease progression $[9,12-14]$. In the CAIRO3 trial, a large randomized trial evaluating maintenance treatment with capecitabin plus bevacizumab, the reintroduction rate of oxaliplatin was $47 \%$ in patients receiving the maintenance therapy [14]. The overall reintroduction rates were reported $40-50 \%$ in several other studies testing intermittent use of oxaliplatin $[8,9$, 11-13]. In our study, oxaliplatin was reintroduced in $55.8 \%$ of the whole cohort, and $64.4 \%$ of patients who received maintenance therapy could tolerate reintroduction, achieving a $28 \%$ objective response and $83 \%$ disease control during the reintroduction phase. In addition, the 22 patients $(43 \%)$ who could accomplish 8 cycles of maintenance therapy had achieved relatively good prognosis, with 15.0 months of PFS and 38.3 months of OS. Although this study was not randomized and the consideration of several biases due to disease biology should be required, these results suggest that our treatment plan could be feasible as an intermitted oxaliplatin treatment strategy.

In the E3200 trial, which investigated the addition of bevacizumab to FOLFOX in 829 patients with previously treated mCRC, the incidence of PSN with grade 3 or higher was significantly higher in the bevacizumab arm, and authors attributed the exacerbation of oxaliplatininduced PSN to a longer duration of chemotherapy or higher cumulative dose in the bevacizumab arm [15]. In our previous study, CCOG-0704 evaluating FOLFOX with an oral fluoropyrimidine maintenance therapy, the median PFS and OS were 7.4 and 28.0 months, respectively, and the incidence of PSN was much higher at 93.3\% [11], although the dose intensity of oxaliplatin was similar to that in the present study. Although the designs of our studies do not allow for a quantified comparison, these results suggest that the addition of bevacizumab to cytotoxic regimens may contribute to survival benefits, and at least may not exacerbate oxaliplatin-induced PSN.

This study has several limitations. First, tumour size and response according to RECIST were not evaluated by central review. Second, the one-arm design and relatively small sample size of this study necessitates confirmation of these results in a larger cohort study. However, it does imply that this strategy, with a brief induction therapy with CapeOX plus bevacizumab, followed by a fixed-term maintenance therapy is feasible for the Japanese patients with mCRC. Data regarding the safety of this strategy was more robust, especially the 

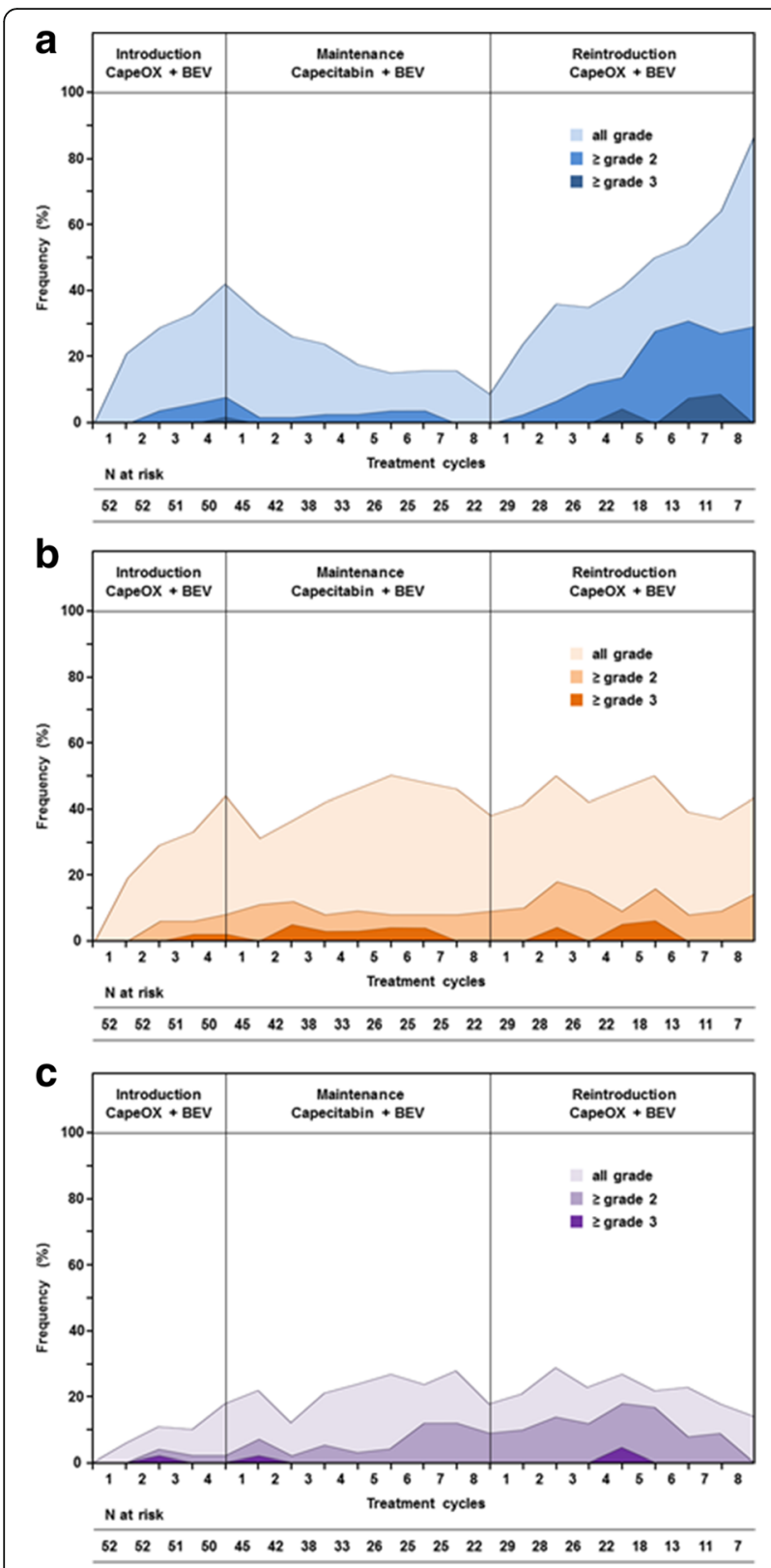

Fig. 3 a. Incidence of peripheral sensory neuropathy. The cumulative peripheral sensory neuropathy rate was $63.5 \%$, including $3.8 \%$ of patients with grade 3 . The frequencies of PNS after 4 cycles of initial CapeOX plus bevacizumab therapy and 8 cycles of the maintenance therapy with capecitabin plus bevacizumab were 42 and 9\%, respectively. CapeOX, capecitabin and oxaliplatin; BEV, bevacizumab; N, total number of patients. $\mathbf{b}$. Incidence of hand-foot syndrome. The frequencies of hand-foot syndrome were $63.5 \%$ in induction therapy, $48.9 \%$ in maintenance therapy, and $55.2 \%$ in reintroduced therapy. CapeOX, capecitabin and oxaliplatin; BEV, bevacizumab; N, total number of patients. $\mathbf{c}$. Incidence of bevacizumab-related toxicities. The frequencies of bevacizumab-related toxicities were $26.9 \%$ in induction therapy, $37.8 \%$ in maintenance therapy, and $37.9 \%$ in reintroduced therapy. CapeOX, capecitabin and oxaliplatin; BEV, bevacizumab; N, total number of patients incidence of PNS. From these encouraging data, it can now be recommended that a randomized controlled trial involving a larger numbers of patients be performed in Japan to obtain more robust and detailed data regarding the efficacy of this strategy and the continued use of bevacizumab.

\section{Conclusion}

In summary, the planned oxaliplatin stop-and-go strategy with a brief induction therapy of CapeOX plus bevacizumab and maintenance therapy of capecitabin plus bevacizumab is feasible for the Japanese mCRC patients.

\section{Abbreviations}

mCRC: Metastatic colorectal cancer; CapeOX: Capecitabine and oxaliplatin; OS: Overall survival; PFS: Progression-free survival; PNS: Peripheral sensory neuropathy; CCOG: Chubu Clinical Oncology Group; RECIST: Response Evaluation Criteria in Solid Tumors; ECOG: Eastern Cooperative Oncology Group; UMIN: University Hospital Medical Information Network; DDC: Duration of disease control; ORR: Overall response rate; CR: Complete response; PR: Partial response; DCR: Disease control rate; SD: Stable disease; NCI-CTC: National Cancer Institute Common Toxicity Criteria; Cl: Confidence interval

\section{Acknowledgments \\ None.}

\section{Funding}

Unrestricted educational grant to CCOG from Chugai Pharmaceutical Co., Ltd.

\section{Availability of data and materials}

The dataset supporting the conclusions of this article is available at request from the corresponding author.

\section{Authors' contributions}

GN participated in study design, served as principal investigator for the clinical trial, and oversaw the data analysis; Kl, HY, KU, HK and Al contributed to protocol conception, provided study data through recruitment of patients, and revised the manuscript; NH and NT contributed to data collection and management, and helped to draft the manuscript; $\mathrm{NH}, \mathrm{DK}$ and $\mathrm{CT}$ provided central coordination, data collection and management, and revised the manuscript; MH, MK, SY, HS, MK and MF contributed to data collection and revised the manuscript; TF performed statistical analysis and helped to draft the manuscript; KM performed statistical analysis and interpretation of data set, and revised the manuscript; YA and YK contributed to conception of study and provided criticism of the manuscript. All authors approved the final manuscript to be published, and agreed to be accountable for all aspects of this study.

\section{Competing interests}

A potential competing interest is study funding provided by an unrestricted educational grant to CCOG from Chugai Pharmaceutical Co., Ltd. The authors declare no other potential competing interests.

\section{Consent for publication}

Not applicable.

\section{Ethics approval and consent to participate}

The study will be being performed in accordance with the Declaration of Helsinki and with all applicable regulatory requirements in Japan directive. The ethics committees in all 18 participating hospitals, including Nagoya University Hospital, Konan Kosei Hospital, Komaki City Hospital, Aichi Cancer Center Aichi Hospital, Kainan Hospital, Tosei Hospital, Tajimi Hospital, Toki General Hospital, Nakatsugawa City Hospital, Okazaki City Hospital, Nagoya Medical Center, Ichinomiya Municipal Hospital, Tohno Kosei Hospital, Chuno Kosei Hospital, Tokai Central Hospital, Yokkaichi City Hospital, Gamagori City Hospital and Toyohashi Medical Center, approved this study. Central Ethics 
approval was obtained by the ethics committee of Nagoya University Hospital (the reference number 218045), Nagoya University Hospital, Nagoya, Japan. The written approval and consent had been obtained before participating in this study.

\section{Publisher's note}

Springer Nature remains neutral with regard to jurisdictional claims in published maps and institutional affiliations.

\section{Author details}

'Department of Gastroenterological Surgery, Nagoya University Graduate School of Medicine, 65 Tsurumai-cho, Showa-ku, Nagoya 466-8550, Japan. ${ }^{2}$ Department of Surgery, Konan Kosei Hospital, Konan, Japan. ${ }^{3}$ Department of Surgery, Komaki City Hospital, Komaki, Japan. ${ }^{4}$ Department of Surgical Oncology, Nagoya University Graduate School of Medicine, Nagoya, Japan. ${ }^{5}$ Department of Gastroenterological Surgery, Aichi Cancer Center Aichi Hospital, Okazaki, Japan. 'Department of Surgery, Okazaki City Hospital, Okazaki, Japan. 'Department of Surgery, Tosei Hospital, Seto, Japan. 'Division of Biostatistics, Clinical Research Center, Aichi Medical University Hospital, Nagakute, Japan. 'Department of Clinical Oncology and Chemotherapy, Nagoya University Hospital, Nagoya, Japan.

Received: 28 June 2016 Accepted: 29 March 2017

Published online: 04 April 2017

\section{References}

1. Saltz LB, Cox JV, Blanke C, Rosen LS, Fehrenbacher L, Moore MJ, et al. Irinotecan plus fluorouracil and leucovorin for metastatic colorectal cancer. N Engl J Med. 2000;343:905-14.

2. Giacchetti S, Perpoint B, Zidani R, Le Bail N, Faggiuolo R, Focan C, et al. Phase III multicenter randomized trial of oxaliplatin added to chronomodulated fluorouracil-leucovorin as first-line treatment of metastatic colorectal cancer. J Clin Oncol. 2000;18:136-47.

3. Martoni AA, Pinto C, Di Fabio F, Lelli G, Rojas L, Limpe FL, et al. Capecitabine plus oxaliplatin (XELOX) versus protracted 5 -fluorouracil venous infusion plus oxaliplatin (pvifox) as first-line treatment in advanced colorectal cancer: a GOAM phase II randomized study (FOCA trial). Eur J Cancer. 2006:42:3161-8.

4. Cassidy J, Clarke S, Díaz-Rubio E, Scheithauer W, Figer A, Wong R, et al Randomized phase III study of capecitabine plus oxaliplatin compared with fluorouracil/folinic acid plus oxaliplatin as first-line therapy for metastatic colorectal cancer. J Clin Oncol. 2008;26:2006-12.

5. Saltz LB, Clarke S, Díaz-Rubio E, Scheithauer W, Figer A, Wong R, et al. Bevacizumab in combination with oxaliplatin-based chemotherapy as firstline therapy in metastatic colorectal cancer: a randomized phase III study. J Clin Oncol. 2008;26:2013-9.

6. Hurwitz H, Fehrenbacher L, Novotny W, Cartwright T, Hainsworth J, Heim W, et al. Bevacizumab plus irinotecan, fluorouracil, and leucovorin for metastatic colorectal cancer. N Engl J Med. 2004;350:2335-42.

7. Hochster HS, Hart LL, Ramanathan RK, Childs BH, Hainsworth JD, Cohn AL, et al. Safety and efficacy of oxaliplatin and fluoropyrimidine regimens with or without bevacizumab as firstline treatment of metastatic colorectal cancer: results of the TREE study. J Clin Oncol. 2008;26:3523-9.

8. Tournigand C, Cervantes A, Figer A, Lledo G, Flesch M, Buyse M, et al. OPTIMOX1: a randomized study of FOLFOX4 or FOLFOX7 with oxaliplatin in a stop-and-go fashion in advanced colorectal cancer-a GERCOR study. J Clin Oncol. 2006;24:394-400.

9. Chibaudel B, Maindrault-Goebel F, Lledo G, Mineur L, André T, Bennamoun $\mathrm{M}$, et al. Can chemotherapy be discontinued in unresectable metastatic colorectal cancer? The GERCOR OPTIMOX2 study. J Clin Oncol. 2009;27:5727-33.

10. Adams RA, Meade AM, Seymour MT, Wilson RH, Madi A, Fisher D, et al. Intermittent versus continuous oxaliplatin and fl uoropyrimidine combination chemotherapy for first-line treatment of advanced colorectal cancer: results of the randomised phase 3 MRC COIN trial. Lancet Oncol. 2011;12:642-53.

11. Nakayama G, Kodera Y, Yokoyama H, Okuda N, Watanabe T, Tanaka C, et al. Modified FOLFOX6 with oxaliplatin stop-and-go strategy and oral S-1 maintenance therapy in advanced colorectal cancer: CCOG-0704 study. Int J Clin Oncol. 2011;16:506-11.
12. Díaz-Rubio E, Gómez-España A, Massutí B, Sastre J, Abad A, Valladares M, et al. First-line XELOX plus bevacizumab followed by XELOX plus bevacizumab or single-agent bevacizumab as maintenance therapy in patients with metastatic colorectal cancer: the phase III MACRO TTD study. Oncologist. 2012;17:15-25.

13. Tezuka T, Hamada C, Ishida H, Ooshiro M, Matsuoka H, Kawasaki S, et al. Phase II clinical study of modified FOLFOX7 (intermittent oxaliplatin administration) plus bevacizumab in patients with unresectable metastatic colorectal cancer-CRAFT study. Investig New Drugs. 2013;5:1321-9.

14. Simkens LH, van Tinteren H, May A, ten Tije AJ, Creemers GJ, Loosveld OJ, et al. Maintenance treatment with capecitabine and bevacizumab in metastatic colorectal cancer (CAIRO3): a phase 3 randomised controlled trial of the Dutch colorectal cancer Group. Lancet. 2015;385:1843-52.

15. Giantonio BJ, Catalano PJ, Meropol NJ, O'Dwyer PJ, Mitchell EP, Alberts SR, et al. Bevacizumab in combination with oxaliplatin, fluorouracil, and leucovorin (FOLFOX4) for previously treated metastatic colorectal cancer: results from the eastern cooperative Oncology Group study E3200. J Clin Oncol. 2007:25:1539-44

\section{Submit your next manuscript to BioMed Central and we will help you at every step:}

- We accept pre-submission inquiries

- Our selector tool helps you to find the most relevant journal

- We provide round the clock customer support

- Convenient online submission

- Thorough peer review

- Inclusion in PubMed and all major indexing services

- Maximum visibility for your research

Submit your manuscript at www.biomedcentral.com/submit
Biomed Central 